\title{
Treatment of relapsed extranodal natural killer/T-cell lymphoma with bortezomib plus fludarabine
}

\author{
$\mathrm{CHEN} \mathrm{CHEN}^{1}$ and HONGMIN $\mathrm{HE}^{2}$ \\ Departments of ${ }^{1}$ Hematology and ${ }^{2}$ Pathology, Zaozhuang Municipal Hospital, Zaozhuang, Shandong 277101, P.R. China
}

Received October 5, 2016; Accepted April 13, 2017

DOI: $10.3892 /$ mco.2017.1364

\begin{abstract}
Extranodal NK/T cell lymphoma (ENKL) is a rare entity, associated with an aggressive clinical behavior and poor prognosis. The optimal treatment strategies, particularly for relapsed patients, have not been clearly determined. A 40-year-old female ENKL patient with orbital involvement as the first presentation was initially suspected to have an orbital pseudotumor. When the patient developed disease progression following treatment with methylprednisolone, biopsy of the orbital mass was performed. Histopathological examination of the biopsy specimen was consistent with the diagnosis of ENKL (Ann Arbor stage IE). The patient was then treated with radiotherapy followed by chemotherapy with dexamethasone, methotrexate, ifosfamide, L-asparaginase and etoposide (SMILE regimen). Despite an initial good response, the patient developed disease relapse and progression (stage IIIE). Subsequently, treatment was switched to a novel regimen comprising bortezomib and fludarabine as salvage therapy for two courses. A positron emission tomography scan revealed disappearance of the orbital tumor. The patient then received autologous hematopoietic stem cell transplantation. At the last follow-up (March 2017), the patient remained disease-free.
\end{abstract}

\section{Introduction}

Extranodal natural killer/T-cell lymphoma (ENKL) is a type of lymphoma associated with Epstein-Barr virus (EBV) infection, which predominantly affects the nasal cavity. However, ENKL with an orbital lesion as the initial presentation is extremely rare, with only a small number of cases reported in the literature to date, as such cases are easily missed or delayed. Currently, the treatment of ENKL is difficult due to its aggressive behavior. Although there is no gold standard for a specific protocol in ENKL, several aspects are widely

Correspondence to: Dr Chen Chen, Department of Hematology, Zaozhuang Municipal Hospital, 41 LongTou Road, Zaozhuang, Shandong 277101, P.R. China

E-mail: ujscc1216@163.com

Key words: extranodal natural killer/T-cell lymphoma, pharmacotherapeutics, bortezomib, fludarabine accepted in the treatment of this disease. Radiotherapy (RT) must be administered alone or in combination with chemotherapy as initial therapy for stage I/II disease; in advanced disease (stage III and IV), asparaginase-based therapy, such as the combination of dexamethasone, methotrexate, ifosfamide, L-asparaginase and etoposide (SMILE regimen), has achieved the best results (1). However, ENKL patients progressing from asparaginase-based chemotherapy represent a challenge. Bortezomib or fludarabine monotherapy has demonstrated considerable efficacy in the treatment of various types of non-Hodgkin lymphoma (NHL). It was demonstrated that the cytotoxicity of bortezomib and fludarabine was synergistic in chronic lymphocytic leukemia cell line models (2), but their efficacy remains unclear in ENKL. To the best of our knowledge, this is the first case report of a relapsed ENKL patient with orbital involvement as the initial presentation treated with RT and the SMILE regimen being successfully salvaged with the combination of bortezomib and fludarabine.

\section{Case report}

A 40-year-old woman presented with a 3-week history of fever and unilateral periorbital edema on the left side. Orbital computed tomography (CT) scans identified a mass in the left orbit, and fine-needle aspiration cytology (FNAC) examination of the mass in another hospital revealed atypical lymphocytic infiltration. The patient was admitted to our hospital in November 2014. On admission, thorough physical examination identified no other positive signs, apart from fever and the abnormality of the left eye. Hematological parameters, thyroid, liver, kidney function and coagulation system tests were all normal. Rheumatoid factor, antinuclear antibody, myeloperoxidase antineutrophil cytoplasmic antibodies and proteinase 3 antineutrophil cytoplasmic antibodies were negative. Serological tests for hepatitis B, Coxsackie virus, cytomegalovirus, influenza virus, antihuman parvovirus B19 and herpes simplex virus were all negative, but the results of the EBV serology were $\operatorname{IgM}^{-}$and $\mathrm{IgG}^{++}$. Blood culture revealed no evidence of bacteremia, and chest radiography revealed no evidence of cardiopulmonary disease or a mediastinal mass. On abdominal ultrasound examination, the liver and spleen were normal, without enlarged lymph nodes.

There was no evidence of sepsis, infectious mononucleosis, Graves' disease or vasculitides. Orbital pseudotumor was suspected based on the CT scans and FNAC examination. 
The patient was treated with methylprednisolone $500 \mathrm{mg} / \mathrm{day}$ for 3 days, and became afebrile, whereas the edema of the orbit significantly regressed. Methylprednisolone was gradually tapered down to $50 \mathrm{mg}$ /day. Orbital edema and fever recurred, without other positive signs on physical examination. Bone marrow cytomorphological examination and biopsy were normal. CT scans identified a mass in the left orbit and increased thickness of the nasal mucosa; the neck, chest, abdomen and pelvis were normal. Bioptic specimens were collected from the orbital mass and the thickened nasal mucosa. Histopathological examination revealed infiltration by atypical lymphoid cells with an angiocentric growth pattern (Fig. 1). Immunohistochemistry of the neoplastic cells was positive for CD2, CD56, CD45RO, cytoplasmic CD3 (Fig. 2), TIA1, granzyme $\mathrm{B}$, perforin and latent membrane protein 1 , and negative for CD5, CD20, CD21 and surface CD3. Moreover, in situ hybridization of the neoplastic cells was positive for EBV-encoded small RNA. The findings supported the diagnosis of ENKL (Ann Arbor stage IE).

The patient was treated with involved-field RT $(50 \mathrm{~Gy})$ followed by 2 cycles of SMILE chemotherapy with growth factor support, repeated every 28 days. A CT scan revealed disappearance of the orbital tumor. Subsequently, the SMILE regimen was applied as maintenance therapy but was complicated by toxicities including grade 4 neutropenia and grade 3 infection. Despite the initial good response, the patient developed disease relapse and progression (stage IIIE) after a total of 4 cycles of SMILE, with hyperpyrexia, painful swelling of the throat and edema of the orbit; the bilateral tonsils protruded from the tonsillar fossa and exhibited an irregular surface. The bilateral cervical, axillary and inguinal lymph nodes were enlarged, with accompanying splenomegaly. A positron emission tomography (PET) scan revealed a mass in the left orbit (Fig. 3A). After obtaining informed consent from the patient, treatment was switched to a novel regimen comprising bortezomib $\left(1.3 \mathrm{mg} / \mathrm{m}^{2}\right.$ on days $1,4,8$ and 11) and fludarabine $\left(25 \mathrm{mg} / \mathrm{m}^{2}\right.$ on days $\left.1-5\right)$ as salvage therapy, repeated every 3 weeks. The hematological toxicities of bortezomib plus fludarabine were well-tolerated, with only grade 3 neutropenia (according to World Health Organization criteria); the non-hematological toxicities were mild. After two courses of chemotherapy, PET scan revealed disappearance of the orbital tumor (Fig. 3B). Subsequently, the patient received autologous hematopoietic stem cell transplantation (HSCT).

\section{Discussion}

ENKL is a lymphoma of cytotoxic lymphocytes characterized by vascular destruction and necrosis. ENKL occurs more commonly in Asia and South America compared with Europe, and predominantly affects the nasal cavity. Other extranasal sites have been occasionally reported, such as the upper aerodigestive tract, gastrointestinal tract, liver, spleen, central nervous system, bone marrow, skin, soft tissue and testis (3). However, ENKL with an orbital lesion as the initial presentation is very unusual, with only a small number of cases reported in the literature. The orbit is a common extranodal site of lymphoid tumors. However, the majority of orbital lymphomas are B-cell lymphomas of mucosa-associated lymphoid tissue (MALT) lymphomas (4), composed mainly of non-active and mature

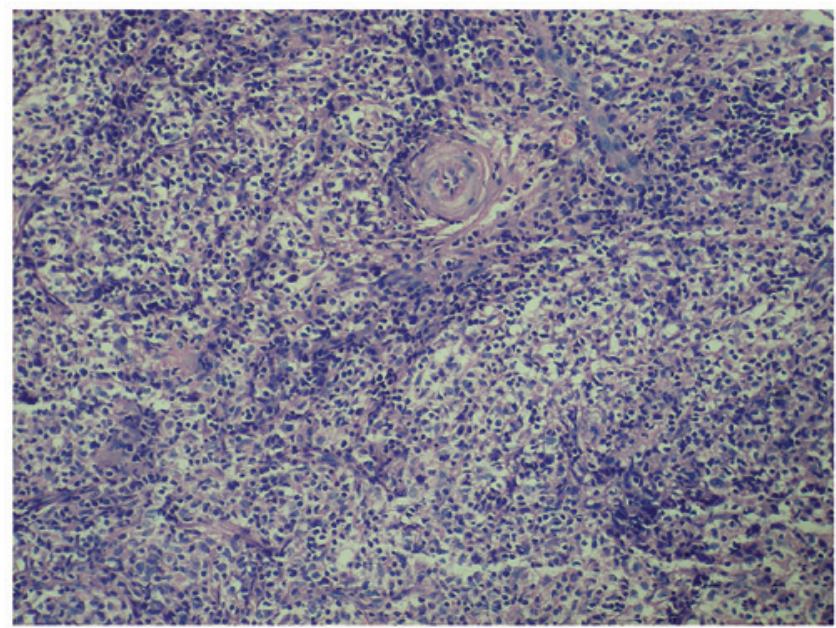

Figure 1. Microscopically, an angiocentric infiltrate of lymphocytes and atypical lymphoid cells was observed. The cells were characterized by pleomorphism, medium to large nuclei and prominent nucleoli (hematoxylin and eosin staining; magnification, $\mathrm{x} 40$ ).

small lymphocytes, often confined to the orbit, and exhibiting low-grade malignant characteristics. Among 68 patients with adnexal lymphomas, 61 were of the MALT type, 2 were of the diffuse large B-cell type, 2 were of the mantle-cell type, 1 was an anaplastic large-cell lymphoma, and only 2 cases were NK/T-cell lymphomas (5). Due to rarity and variable clinical manifestations, ENKL with orbital involvement may be easily misdiagnosed. In the early stages of the disease, relatively few neoplastic cells are present, and the neoplastic cells are usually located angiocentrically, with areas of surrounding necrosis. Thus, biopsy may not include the tumor cells. Due to these factors, multiple or extensive biopsies are often required to obtain sufficient viable tissue. Atypical manifestations, including swollen periorbital tissues, high orbital pressure and edematous skin of the eyelids and the inner canthus, the rapid progression of the disease and massive tissue necrosis may lead to the misdiagnosis as an acute orbital pseudotumor, such as our patient. Orbital ENKL is most often misdiagnosed as orbital cellulitis and thyroid ophthalmopathy (6,7). In addition, conditions that may cause or mimic ENKL with orbital involvement also include lymphangioma, metastatic carcinoma and rheumatological disorders, such as Wegner's granulomatosis.

Although optimal treatment strategies for ENKL have not been clearly determined, several aspects are widely accepted in the treatment of this disease. For localized disease, RT must be administered alone or in combination with chemotherapy as initial therapy. In disseminated NK/T-cell lymphomas, systemic chemotherapy is the mainstay of treatment. Allogeneic HSCT has also been evaluated in the management of ENKL in several retrospective patient series, but the results are difficult to interpret, as the majority of the studies included a small number of cases, with heterogeneous indications and inclusion criteria (8-10). ENKL is radiosensitive, and RT alone ( $\sim 50 \mathrm{~Gy}$ ) has achieved overall response rates of $77-100 \%$ for localized disease. However, RT alone is inadequate, with reported systemic relapse rates of $25-40 \%$, and chemotherapy is required to decrease the risk of systemic failure (11). A multicenter retrospective study reported that, in 36 patients with ENKL (nasal type), the use of RT with 


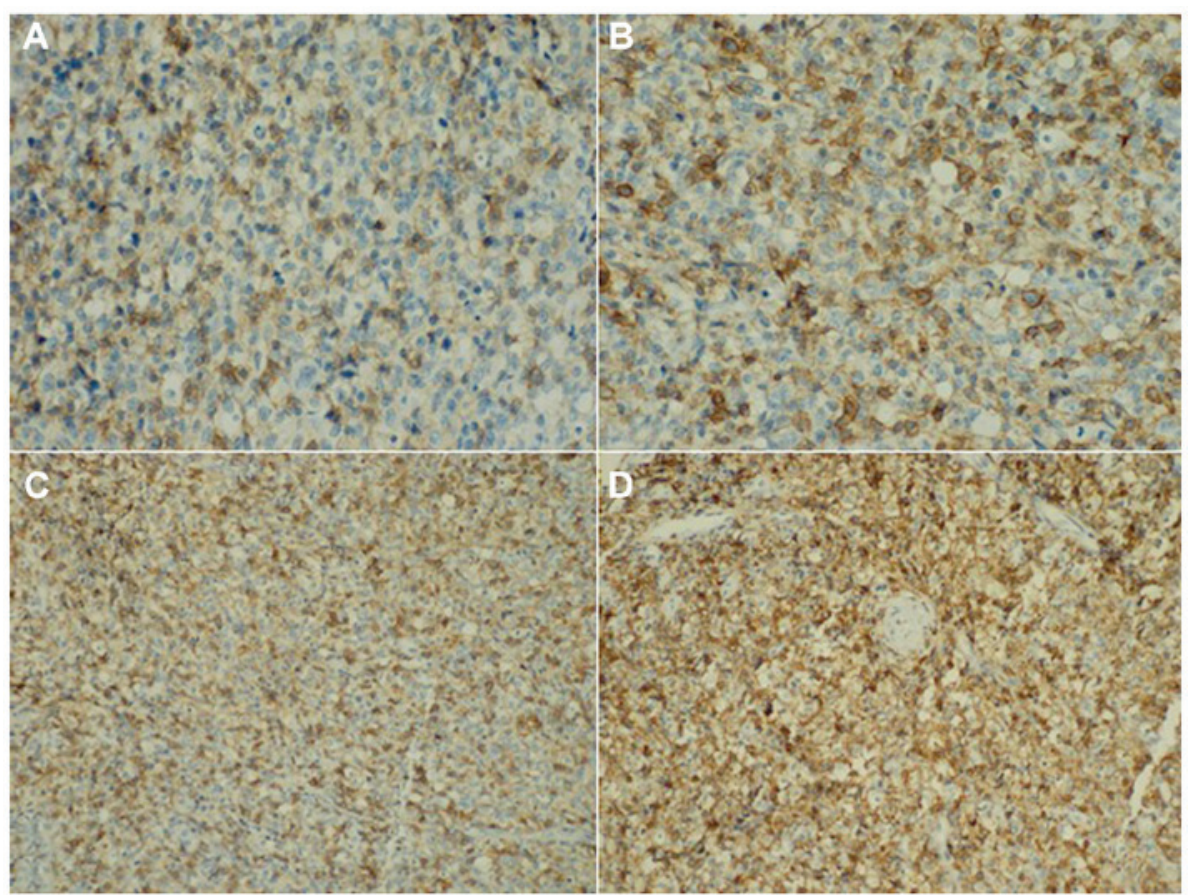

Figure 2. Immunohistochemistry. The neoplastic cells were positive for (A) CD2 (magnification, x200), (B) CD56 (magnification, x200), (C) CD45RO (magnification, x100) and (D) cytoplasmic CD3 (magnification, x100).
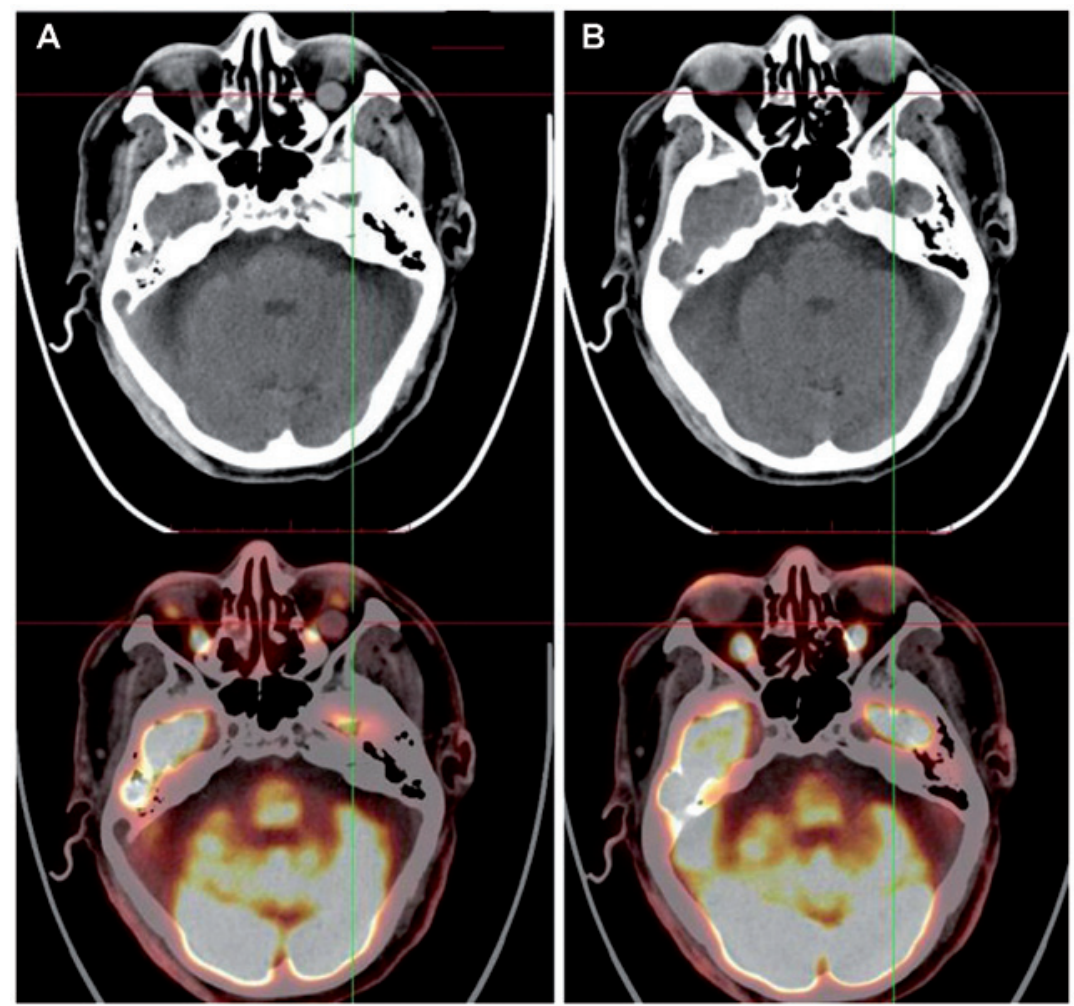

Figure 3. Images of positron emission tomography (A) before and (B) after treatment with bortezomib in combination with fludarabine.

chemotherapy (either concurrent or sequential) compared with chemotherapy alone was associated with significantly increased complete response (CR) rate (90 vs. 33\%, respectively) and a higher 5 -year overall survival (OS) rate (75 vs. $35 \%$, respectively) (12). Anthracycline-based regimens (such as CHOP) followed by involved-field RT yielded unsatisfactory response rates. Kim et al used 4 cycles of CHOP chemotherapy followed by involved-field RT ( $45 \mathrm{~Gy}$ ) for stage I/II nasal lymphoma, with a CR rate of $58 \%$ and a 3 -year OS rate of 59\% (13). The poor response to CHOP may be due to the intrinsic properties of the ENKL. Generally, NK cells overexpress P-glycoprotein (14), which results in drug efflux and 
intracellular decrease of cytotoxic agents. SMILE is one of the most promising protocols, comprising L-asparaginase, 3 non-P-glycoprotein-dependent drugs (dexamethasone, methotrexate and ifosfamide) and etoposide, which has been tested in a multicenter phase 2 study conducted by the NK Tumor Study Group, where 38 patients with stage IV, relapsed/refractory ENKL were treated, and the results showed a CR rate of $45 \%$ and a PR rate of $34 \%$ (15). Kwong et al treated 43 newly diagnosed and 44 relapsed/refractory ENKL patients with the SMILE regimen, with an overall response rate of $81 \%$ (CR rate of $66 \%$ and partial response rate of $15 \%$ ), and the response rates were similar between newly diagnosed and relapsed/refractory patients. At a median follow-up of 31 months, the 5-year OS was $50 \%$ and the 4-year disease-free-survival rate was $64 \%$ (16). However, the management of patients with ENKL progressing while on asparaginase-based chemotherapy represents a challenge.

ENKL cells constitutively express nuclear factor (NF)- $\mathrm{kB}$, and NF- $\kappa \mathrm{B}$ is associated with oncogenesis, cell cycle progression, apoptosis and multiple drug resistance. Bortezomib is a dipeptidyl boronic acid that is a specific and selective inhibitor of the $26 \mathrm{~S}$ proteasome, which leads to stabilization of IкB $\alpha$, an NF- $\kappa \mathrm{B}$ inhibitory protein, thereby reducing NF- $\kappa \mathrm{B}$ activity. Moreover, bortezomib may act by inhibiting DNA repair kinases (17), supporting the use of bortezomib in combination with cytotoxic agents. Currently, the clinical efficacy of bortezomib for hematological neoplasms is an interesting issue. A phase I study of bortezomib plus CHOP in patients with advanced, aggressive T-cell or NK/T-cell lymphoma demonstrated that 1 in 3 patients achieved CR (18). Fludarabine, a purine nucleoside analogue, is a potent cytotoxic agent that acts by inhibiting DNA polymerase and ribonucleotide reductase, thus terminating DNA strand replication. Clinical studies have demonstrated that fludarabine (alone or, particularly, as a component of combination therapy) may result in high overall response rate and CR rate in adults with various types of NHL (19). However, the clinical efficacy of fludarabine has not been well estimated in ENKL.

It was demonstrated that the cytotoxicity of fludarabine and bortezomib was synergistic in chronic lymphocytic leukemia cell line models (2). The potential synergistic mechanism may involve inhibiting the repair of the fludarabine-induced DNA lesions by bortezomib, thereby enhancing its antineoplastic effect. Moreover, another study also suggested that bortezomib may reverse resistance to fludarabine by inhibiting the NF- $\mathrm{KB}$ pathway (20). Hence, it was hypothesized that chemotherapy with bortezomib plus fludarabine may be an effective salvage strategy for relapsed ENKL. After obtaining informed consent from the patient, bortezomib in combination with fludarabine was administered as salvage treatment, which achieved a promising result.

The present case suggests that bortezomib plus fludarabine appears to be a safe and effective alternative for relapsed ENKL. However, the efficacy of this combination should be evaluated in further larger-sized clinical trials.

\section{References}

1. Tse E and Kwong YL: How I treat NK/T-cell lymphomas. Blood 121: 4997-5005, 2013.
2. Duechler M, Linke A, Cebula B, Shehata M, Schwarzmeier JD, Robak $\mathrm{T}$ and Smolewski P: In vitro cytotoxic effect of proteasome inhibitor bortezomib in combination with purine nucleoside analogues on chronic lymphocytic leukaemia cells. Eur J Haematol 74: 407-417, 2005.

3. Vega F, Lin P and Medeiros LJ: Extranodal lymphomas of the head and neck. Ann Diagn Pathol 9: 340-350, 2005.

4. Ferry JA, Fung CY, Zukerberg L, Lucarelli MJ, Hasserjian RP, Preffer FI and Harris NL: Lymphoma of the ocular adnexa: A study of 353 cases. Am J Surg Pathol 31: 170-184, 2007.

5. Cho EY, Han JJ, Ree HJ, Ko YH, Kang YK, Ahn HS, Ahn SD, Park CJ and Huh J: Clinicopathologic analysis of ocular adnexal lymphomas: Extranodal marginal zone b-cell lymphoma constitutes the vast majority of ocular lymphomas among Koreans and affects younger patients. Am J Hematol 73: 87-96, 2003.

6. Charton J, Witherspoon SR, Itani K, Jones FR, Marple B and Morse B: Natural killer/T-cell lymphoma masquerading as orbital cellulitis. Ophthal Plast Reconstr Surg 24: 143-145, 2008.

7. Dai W, Zhong M, Shen W, Zou K and Bai CG: Natural killer T-cell lymphoma originating from the orbit. Chin Med J (Engl) 125: 1677-1680, 2012.

8. Ennishi D, Maeda Y, Fujii N, Kondo E, Shinagawa K, Ikeda K, Ichimura K, Yoshino T and Tanimoto M: Allogeneic hematopoietic stem cell transplantation for advanced extranodal natural killer/T-cell lymphoma, nasal type. Leuk Lymphoma 52: 1255-1261, 2011.

9. Kwong YL: Hematopoietic stem cell transplantation in natural killer cell lymphoma and leukemia. Int J Hematol 92: 702-707, 2010.

10. Li M, Gao C, Li H, Wang Z, Cao Y, Huang W, Li X, Wang S, Yu L and Da W: Allogeneic haematopoietic stem cell transplantation as a salvage strategy for relapsed or refractory nasal NK/T-cell lymphoma. Med Oncol 28: 840-845, 2011.

11. Kim SJ and Kim WS: Treatment of localized extranodal NK/T cell lymphoma, nasal type. Int J Hematol 92: 690-696, 2010.

12. Chauchet A, Michallet AS, Berger F, Bedgedjian I, Deconinck E, Sebban C, Antal D, Orfeuvre H, Corront B, Petrella T, et al: Complete remission after first-line radio-chemotherapy as predictor of survival in extranodal NK/T cell lymphoma. J Hematol Oncol 5: 27, 2012.

13. Kim WS, Song SY, Ahn YC, Ko YH, Baek CH, Kim DY, Yoon SS, Lee HG, Kang WK, Lee HJ, et al: CHOP followed by involved field radiation: Is it optimal for localized nasal natural killer/T-cell lymphoma? Ann Oncol 12: 349-352, 2001.

14. Wang B, Li XQ, Ma X, Hong X, Lu H and Guo Y: Immunohistochemical expression and clinical significance of P-glycoprotein in previously untreated extranodal NK/T-cell lymphoma, nasal type. Am J Hematol 83: 795-799, 2008.

15. Yamaguchi M, Kwong YL, Kim WS, Maeda Y, Hashimoto C, Suh C, Izutsu K, Ishida F, Isobe Y, Sueoka E, et al: Phase II study of SMILE chemotherapy for newly diagnosed stage IV, relapsed, or refractory extranodalnatural killer (NK)/T-cell lymphoma, nasal type: The NK-Cell Tumor Study Group study. J Clin Oncol 29: 4410-4416, 2011.

16. Kwong YL, Kim WS, Lim ST, Kim SJ, Tang T, Tse E, Leung AY and Chim CS: SMILE for natural killer/T-cell lymphoma: Analysis of safety and efficacy from the Asia Lymphoma Study Group. Blood 120: 2973-2980, 2012.

17. Hideshima T, Mitsiades C, Akiyama M, Hayashi T, Chauhan D, Richardson P, Schlossman R, Podar K, Munshi NC, Mitsiades N and Anderson KC: Molecular mechanisms mediating antimyeloma activity of proteasome inhibitor PS-341. Blood 101: 1530-1534, 2003.

18. Lee J, Suh C, Kang HJ, Ryoo BY, Huh J, Ko YH, Eom HS, Kim K, Park K and Kim WS: Phase I study of proteasome inhibitor bortezomib plus CHOP in patients with advanced, aggressive T-cell or NK/T-cell lymphoma. Ann Oncol 19: 2079-2083, 2008.

19. Anderson VR and Perry CM: Fludarabine: A review of its use in non-Hodgkin's lymphoma. Drugs 67: 1633-1655, 2007.

20. Hewamana S, Alghazal S, Lin TT, Clement M, Jenkins C, Guzman ML, Jordan CT, Neelakantan S, Crooks PA, Burnett AK, et al: The NF-kappaB subunit Rel A is associated with in vitro survival and clinical disease progression in chronic lymphocytic leukemia and represents a promising therapeutic target. Blood 111: 4681-4689, 2008. 